in my experience, tired women and those whom we sometimes call neurasthenics rarely show the sub-normal even during the periods of prolonged rest.

Of the other forms of tuberculosis, especially tuberculous peritonitis may or may not have sub-normal temperature. In the cases I have followed, I have not been impressed with its frequency as a special sign to be depended upon in diagnosis.

Cases of bronchiectasis and fibroid phthisis, and cavity formation after non-tubercular infections, all of these interfering with the action of the lung, can give rise to prolonged sub-normal conditions; but these are not the cases where this range of temperature comes into active play as a help in diagnosis.

Dr. B. G. R. Williams of Paris, Ill., has reported that he had observed a form of "grip" where there was a persistence of temperature of from 94 degrees to 97 degrees, with marked depression. In the cases usually called "grip" or "influenza," my general experience has been that the return to normal has been moderately rapid. In correspondence with Dr. Williams, he states that he has not noticed so far that the cases in which he has noted sub-normal temperature have developed active tuberculosis.

It goes without saying that in doubtful cases everything must be taken into consideration when one sees the patient and makes his diagnosis. Cardiac and renal conditions must not be allowed to escape notice and must be surely eliminated before the sub-normal temperature alone assumes special importance; and then it must be held in mind that there is the possibility that convalescence from various bacterial infections may be found to have periods of subnormal temperature, which must make us careful in our diagnosis; although personally, I do not believe that they enter into consideration to any great extent. And I say this in spite of the statement in Krehl's Clinical Patholog, that "Sub-normal temperatures are more common than is generally supposed. They are often seen during convalescence from infectious diseases; and in such instances, they are generally due to a diminished production of heat with an inefficient heat regulation." (Hewlett's translation.)

\section{COCCYGODYNIA: CAUSES AND TREATMENT.}

\section{By Raipit Dumy, A.B., M.D., Tampa, Fromida.}

Coccygodynia is not a common disorder nor a very grave one, but it is one which may cause great suffering and considerablo disability. I should like in this paper to discuss the condition briefly, and to cite one case, with my method of treatment.

In the first place I should like to differentiate those vague and indefinite pains around the region of the coccyx which we see in various nervous conditions, from true coceygodynia. In neurasthenia often, in tabes at times, in hysteria, and in traumatic neurosis pains in the sacral and coccygeal region are complained of. But such pains are indefinite, they are dull rather than sharp, and there is not much tenderness of the coccyx. The same may be said of the reflex or neuralgic pains in the coccyx encountered in pelvic or anal disease, especially in the female.

True coccygodynia, however, though it has no definite known pathology, has very definite symptoms and signs. The pain is always sharp and lancinating and caused by movements of the body or anus, or by pressure. As long as the patient is still in bed and lying on the side, there is no discomfort. Again, the coccyx is always exquisitely tender on rectal palpation. The pain, too, is definitely located in the coccyx and does not radiate.

Etiology. I will venture the statement that true coccygodynia is always of traumatic origin; at least that has been my observation in the cases 1 have seen. The trauma is either external-falls, kicks or blows, or intermal-in childbirth. It seems to me that the etiology which best explains the symptoms is injury to the sacrococeygeal articulation or to the nerves in and around that articulation, this injury causing chronic inflammatory change. Fracture or caries of the coccyx may account for some cases. Some writers contend that subluxation of the coccyx can always be demonstrated (Hamant and Pigache ${ }^{1}$ ).

Reflex coccygodynia is much more common in women than in men due to pelvic trouble. It may be said hardly to occur in the male. True coccygodynia however occurs in both sexes. It is inuch more common in females, however, due possibly to injuries to the coccyx in parturition. It has been observed at all ages, even in little girls.

Symptoms and Diagnosis. The chief symptom as said before is sharp pain in the coccyx, occurring with pressure, with movements of the legs, or with defecation, that is, with any movement of the coccyx. The muscles attached to the coccyx are the gluteus maximus, in small part, which contracts on any movement of the leg, and the levator ani, sphincter ani, and coccygeus which contract during defecation. The patients often present a very woe-begone appearance. The obstinate character of the pain drains health and nerves. Neurasthenia of vary. ing grades is a not uncommon result. Owing to pain in defecation, the patients refrain from going to stool as far as possible, and constipation is the natural consequence. The chief and indeed the only physical sign in most cases is extreme tenderness of the coccyx on rectal palpation. Pinching the coccyx between the rectal finger and thumb causes the patient to wince with pain. Deviations of the coccyx, subluxa- 
tion, and infiltration in the region of the sacrococcygeal articulation are mentioned by some writers. The diagnosis should present no especial difficulty. The rectum and pelvis should be examined for local lesions (hemorrhoids, fissure, retrodisplacement, etc.) which might account for reflex pain in the coccyx. Spinal cord lesions and sciatica must be excluded.

Treatment. Nervous or reflex coccygodynia calls for appropriate treatment of the underlying condition, along with local remedies. Radical surgical treatinent is out of place. For true coccygodynia medical treatment is only temporizing. There are two forms of surgical treatment. The first is excision of the coccyx. Most writers from Nott ${ }^{2}$,who performed the first excision, on, testify to the efficacy of this operation. Simpson ${ }^{3}$ advocated section of the muscle-insertions on the coceyx, but this plan was discarded as being unsatisfactory. Whitehead ${ }^{4}$, Odell $^{5}$, of early writers, and Fullerton ${ }^{\mathbf{6}}$, more recently, advocate the method of excision.

Excision will probably cure every case of true coccygodynia, if the assumption that the lesion is in the bone or the sacrococcygeal articulation be correct. It will not cure reflex or neuralgic coccygodynia. The operation is extremely simple. A linear incision is made over the coccyx and the end of the sacrum, and with rongeur and elevator the coccyx is disjointed and peeled out. The only drawback to the operation is that the muscle insertions in the coccyx are of value to the perineum, so that the loss of the coccyx weakens that structure.

The other form of surgical treatment is the injection of alcohol. De Vésian ${ }^{7}$, in 1907, first used this method in an obstinate case of reflex coccygodynia, getting the idea from the treatment of facial neuralgia by the injection of alcohol.

De Vésian's case was that of a woman of 43 . There was no history of injury to the coccyx, external or obstetrical. There were inflammatory masses in the pelvis, which De Vésian considered the cause of the coccygodynia. Operation was refused by the patient. Under local treatment the pelvic condition ameliorated, but the pain in the coccyx was still very severe.

After trying other forms of local treatment for the coccygodynia, De Vésian determined to try the injection of alcohol. He used $60 \%$ alcohol. With the index finger of the left hand in the rectum, the needle was inserted back of the rectum and anterior to the coccyx. At the depth of $3 \mathrm{~cm}$., 2 c.c. of alcohol were injected along the sacrococcygeal articulation and the needle withdrawn. Two cubic centimeters of the alcohol were then injected along the posterior surface of the coccyx. But one injection was necessary and the relief was immediate. The patient was under observation for four months, during which time she had no pain.

F. C. Yeomans ${ }^{8}$ has treated seven eases hy this method. His technic is as follows: with the patient on the left side, the right index finger in the rectum determines the point of greatest tenderness. This, he has found, is just below the tip of the coccyx. The needle of the syringe is introduced to this point of maximum tendermess, and 10 to 20 minims of $80 \%$ alcohol are injected slowly. The pain is severe but lasts but a few minutes.

Yeomans has found in general three to five injections necessary for a cure. The interval is from three to five days. He reports no recurrence, the time of observation varying from one month to four years.

In the past two year's I have had several opportunities to use Schlosser's treatment for neuralgia. I have in that time seen but one case of coccygodynia. This case was treated by me with alcohol injections, so far (eight months) with no return of the pain. The case was as follows :

Mrs. Marry M.; aged 33, in September, 1913, fell from the hammock to the porch (a fall of probably 18 inches) striking the sacrum against a child's toy. The patient says the pain was not during the ensuing days extremcly severe. She was not at any time compelled to keep her bed. Several weeks after the injury, the pain became sharper and localized in the region of the coceyx. It has persisted up to the time of observation (Feb. 11, 1914), and is severe enough to be a constant source of distress. Examination shows no pelvic or rectal disease, except a slight laceration of the perineum. The coccyx seems abnormally movable and bent forward, but not apparently dislocated. It is exquisitely tender to palpation. No treatment has been insti-. tuted except salves and liniments.

Having done almost all my minor rectnl and perineal work in the last two years under novocain, it occurred to me to infiltrate the anterior surface of the coccyx with that drug before using the alcohol. The patient was placed on her back in an exaggerated lithotomy position, using Clover's crutch. The index finger was inserted in the rectum for a guide, the whole anterior surface of the eoccyx was then infiltrated with 10 c.c. of $1 \%$ novocain solution with suprarenin. Leaving the needle in situ, the syringe was then partially filled with $80 \%$ alcohol, and 4 c.c. injected along the sacrococcygeal articulation, and the needle withdrawn.

There was considerable soreness for several days following the injection. This gradually disappeared and with it the pain. This patient has been seen by mo several times in the last eight months. She says she feels well and has no pain.

'The alcohol treatment is so simple that it can be employed by any practitioner. It can be used in any case of coccygodynia, independent of its origin, and has this great advantage, that it offers promise of relief in those cases of nenralgic coccygodynia which resist excision.

BHBHOGRAPHY.

1 IJanınt and Pigache: Revue de Chirurgie, Jan., 1814, p. 01. 2 Nott: New Orleans Med. Jour., May, 1844.

- Simpson: Edin. Med. Jour., July, 1801.

- Whitehead: Isancet, July, 1886.

- Odel]: Inncet, Mny 28, 1887.

a kelly-Noble: (Yvnecology, vol. 1, p. 343

7 Do Vesian: Revue Prutique de Gynocologie, d' Obstetrique, et. de Pediatric, duly, 1007 .

* Yeom:ats: Mell. Becord, Aug. 22, 1914. 